\title{
E-Textile RF Energy Harvesting and Storage using Organic-Electrolyte Carbon-Based Supercapacitors
}

\author{
Mahmoud Wagih ${ }^{1}$, Member, IEEE,, Nicholas Hillier ${ }^{2}$, \\ Alex S. Weddell ${ }^{3}$, Member, IEEE, and Steve Beeby ${ }^{4}$, Senior Member, IEEE \\ School of Electronics and Computer Science, University of Southampton, Southampton, United Kingdom \\ $\left\{{ }^{1}\right.$ mahmlg15, ${ }^{3}$ asw, $\left.{ }^{4} \mathrm{spb}\right\} @$ ecs.soton.ac.uk; ${ }^{2}$ nh3g09@ soton.ac.uk
}

\begin{abstract}
Wearable radio frequency (RF) energy harvesting is highly dependent on the distance from the source and human-caused RF shadowing. Therefore, energy storage devices integrated with rectennas are of paramount importance to overcome this intermittency. In this paper, the use of carbonbased e-textile supercapacitors for storing the RF-DC converted power for powering body area networks nodes is investigated. A voltage doubler sub-1 GHz flexible rectifier, whose peak power conversion efficiency (PCE) approaches $80 \%$ is coupled to a two-cell $15.5 \mathrm{mF}$ textile-based supercapacitor operating up to $4 \mathrm{~V}$ DC. Owing to the rectifier's low optimum load resistance and high DC Voltage output, the average charging PCE of the rectifier-supercapacitor module reaches $31 \%$ for a $9.5 \mathrm{dBm}$ input. Time-varying s-parameter measurements are performed to compare the time-averaged matching as opposed to instantaneous measurements using a resistive load, where the textile supercapacitor exhibits a similar response to a commercial supercapacitor. Finally, the RF-charged textile supercapacitor is demonstrated, for the first time, powering a microcontroller and Bluetooth transmitter with an average power consumption of $350 \mu \mathrm{W}$ for up to $102 \mathrm{~s}$, following $40 \mathrm{~s}$ of charging at $9.5 \mathrm{dBm}$, demonstrating its suitability for RF-powered body area networks applications.
\end{abstract}

Index Terms-Antenna, rectifier, rectenna, supercapacitor, wireless power transmission

\section{INTRODUCTION}

Radio Frequency (RF)-powered body area networks (BANs) have attracted significant research interest, with several textile-based rectenna implementations based on a variety of materials and targeting different frequency bands [1]-[4]. Compared to other forms of energy harvesting, RF energy harvesting rectennas, and more generally RF passives, can be implemented using standard low-cost conductors on most substrates without the need for specific materials [5]. For instance, multi-port RF energy harvesting and communication antennas have been realized using conventional 3D printed substrates [6] as well as all-fabric antennas [7].

Despite their high efficiency, low cost, and ease of construction, wearable RF energy harvesting rectennas suffer from a high intermittency due to the mobility of the user, and body-induced shadowing. It was previously found that, when considering the average angular gain of a wearable rectenna, the harvested power could drop by at least 50\% [8]. As a

This work was supported by the UK Engineering and Physical Sciences Research Council (EPSRC) under Grants EP/P010164/1 and EP/L016818/1. result, the integration of a wearable rectenna with a textile supercapacitor was proposed to overcome the transient nature of microwave power transmission to wearables [1].

Compared to previous implementations where rectennas were integrated with capacitors [9], supercapacitors [10], or batteries [11], the e-textile module in [1] achieved the highest average wireless charging power conversion efficiency (PCE) of up to $37 \%$. While this highlights the feasibility of highefficiency RF harvesting and storage using e-textile materials, at least three supercapacitor cells were required to reach a voltage level around $3 \mathrm{~V}$, which falls below the $3.3 \mathrm{~V}$ threshold of several commercial devices, as well as below the maximum voltage output of voltage-multiplying rectifiers which can surpass $8 \mathrm{~V}$ [1]. Therefore, there is a need for improved textile-based supercapacitors, compatible with the voltage operation range of typical BAN nodes and with the output of state-of-the-art rectennas [1].

In this paper, the integration of an organic-electrolyte carbon-based e-textile supercapacitor [12] with a sub-1 GHz rectifier is investigated. Using an improved electrolyte over our previous rectenna-supercapacitor module [1], the proposed module requires fewer supercapacitor cells to reach the operational voltage of a real sensor node. In Section II, the design and characterization of the rectifier and supercapacitor are presented. We then demonstrate the integrated module and characterize it using time-varying s-parameters, in Section III, before demonstrating it, for the first time, powering a real wireless sensor node load.

\section{E-TEXTILE RECTIFIER AND SUPERCAPACITOR}

\section{A. Rectifier Design and Characterization}

The rectifier used in this work is a coplanar waveguide (CPW) single-stage voltage doubler based on the Infineon BAT15-04R diode, whose breakdown voltage of $4 \mathrm{~V}$ enables up to $8 \mathrm{~V}$ DC output and a high peak PCE around $10 \mathrm{dBm}$ [1]. The rectifier is matched using a single $22 \mathrm{nH}$ lumped inductor. Fig. 1 shows the schematic of the full system, including the inductive-matched $\mathrm{CPW}$ voltage doubler rectifier.

The rectifier was characterized using a Vector Network Analyzer (VNA) as a continuous wave $(\mathrm{CW})$ generator to characterize its DC output, PCE, and reflection coefficient $\left(S_{11}\right)$. In Fig 2(a), it can be observed that the rectifier achieves a peak PCE of $80 \%$ from a $10 \mathrm{dBm}$ input. Due to the relatively 


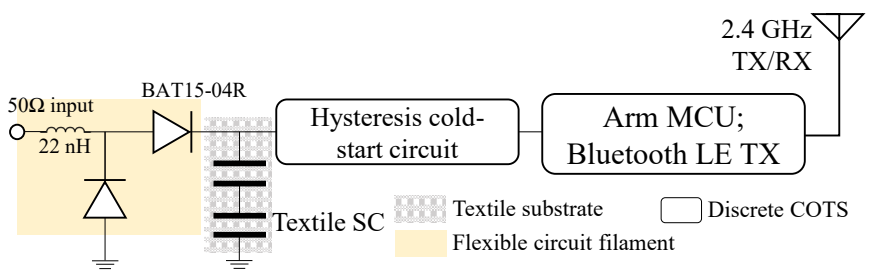

Fig. 1. Schematic of the integrated system, showing the CPW polyimide rectifier and fully-textile supercapacitor, the cold-start circuit, and wireless BAN node load.
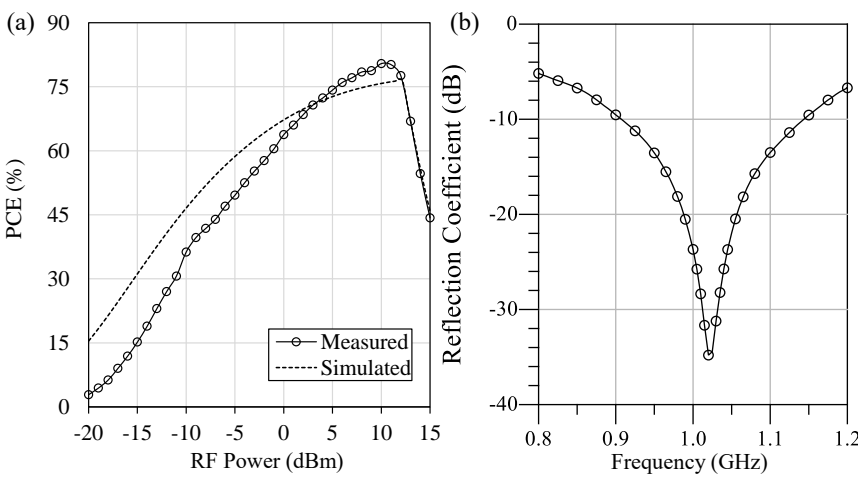

Fig. 2. (a) Simulated and measured DC output and PCE of the voltage doubler rectifier across a $5 \mathrm{k} \Omega$ load at $1 \mathrm{GHz}$, (b) the measured $S_{11}$ response at $10 \mathrm{dBm}$.

high capacitance of the SOT23-packaged diodes, it can be observed that the $22 \mathrm{nH}$ inductor and the low-impedance load (3 $\mathrm{k} \Omega$ ) result in over $200 \mathrm{MHz} S_{11}<-10 \mathrm{~dB}$ bandwidth, as shown in Fig. 2(b). As previously demonstrated in [1], the inductive-matched CPW voltage doubler can produce up to $8 \mathrm{~V}$ DC output voltage.

The CPW rectifier is directly connected to a textile carbonbased supercapacitor utilizing an organic gel electrolyte. By eliminating the power conversion and management stage, the components count can be significantly reduced making it more suitable for integration in wearables and other low-cost flexible and conformable systems [1].

\section{B. Supercapacitor Fabrication}

The supercapacitor is fabricated using spray-coated carbon on a standard textile substrate, with an organic gel electrolyte. The electrodes' ink (9:1 ratio activated carbon to carbon black) was deposited onto a plain weave cotton substrate via an airbrush following the method in [13]. The textile was clamped within an aluminium mask, with $1 \mathrm{~cm}$ diameter $\left(0.785 \mathrm{~cm}^{2}\right)$ holes exposed. The airbrush was then set up $7.5 \mathrm{~cm}$ away from the mask with a pressure of $20 \mathrm{psi}$ $(138 \mathrm{kPa})$ and the first electrode deposited. The mask was then turned around and the second electrodes deposited directly opposite the first, forming a single-layer symmetric supercapacitor with the textile forming the separator layer. The supercapacitors were then cured at $100^{\circ} \mathrm{C}$ for 10 minutes. After this cure, the supercapacitors were weighed and found to have an electrode loading of approximately $2 \mathrm{mg}$ each.

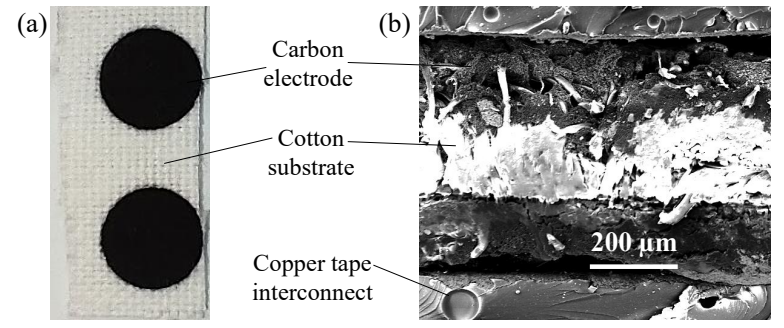

Fig. 3. (a) Photograph of the supercapacitor cells; (b) SEM micrograph of the capacitor's cross-section.

Following the curing process, a $1 \mathrm{M}$ organic gel electrolyte was produced by dissolving $1.63 \mathrm{~g}$ of tetraethylammonium tetrafluoroborate and $0.187 \mathrm{~g}$ of polyacrylamide in $7.5 \mathrm{ml}$ of dimethyl sulfoxide. The supercapacitors were then submerged in the electrolyte before being placed in a vacuum of $25 \mathrm{mbar}$ for 30 minutes to improve the wetting of the electrodes and to remove any trapped air. Fig. 3 shows a photograph of the two-cell textile supercapacitor (a), and a scanning electron microscopy (SEM) cross-section of the individual cell (b).

\section{Integrated Module Characterization}

The time-averaged power conversion efficiency of the rectifier-supercapacitor module is given by

$$
\mathrm{PCE}_{\text {Average }}=\frac{C V^{2}}{2} \times \frac{1}{t} \times \frac{1}{P_{\mathrm{RF}}},
$$

where $C$ is the capacitance of the two-cell textile supercapacitor, characterized to be $15.5 \mathrm{mF}$ at a charge/discharge current density of $0.5 \mathrm{~mA} . \mathrm{cm}^{-2}, V$ is the measured DC potential, $t$ is the charging period, and $P_{\mathrm{RF}}$ is the input power [14].

Based on the $4 \mathrm{~V}$ turn-on voltage of the system, it was found that a minimum of $4.5 \mathrm{dBm} \mathrm{RF}$ input is required. Based on the measured charging time, $\mathrm{PCE}_{\text {Average }}$ was calculated using (1) at 4.5 and $9.5 \mathrm{dBm}$ to be 29.5 and $31 \%$, respectively. The lower average charging efficiency than the instantaneous PCE, in Fig. 2(a), was widely reported [1], [9], [14], and is attributed to the $S_{11}$ time variation, introduced by the varying charging current of the supercapacitor [1]. A VNA was used to measure the time-varying s-parameter of both the proposed textile supercapacitor and a commercial supercapacitor. The measured response in Fig. 4 demonstrates that while the $S_{11}$ for the optimal load is under $-30 \mathrm{~dB}$, the capacitor's charging current varies the observed $S_{11}$. Nevertheless, the omission of a standalone power management circuit along with the designed rectifier result in a higher charging efficiency than previous systems utilizing DC-DC converters [9], [11].

As shown in Fig. 1, the textile-based rectifier and supercapacitor are used to intermittently power an example BAN node. The cold-start circuit is based on two XC61C voltage monitors and a latch switch MOSFET. This creates a hysteresis switching loop enabling the supercapacitor to discharge through the load for $1.6<V_{C}<4.1 \mathrm{~V}$. The load is based on a Texas Instruments CC2640R2F system-on-chip (SoC), comprising a $48 \mathrm{MHz}$ Arm Cortex-M3 microcontroller 


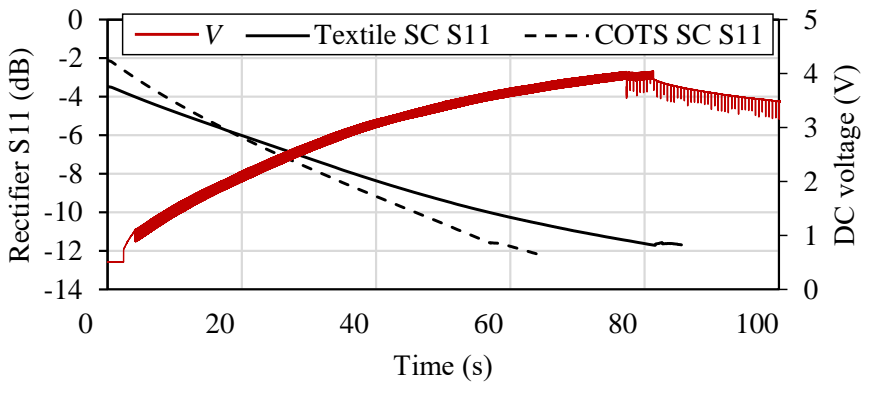

Fig. 4. Measured $S_{11}$ of the rectifier while charging the textile supercapacitor and an off-the-shelf (COTS) supercapacitor.

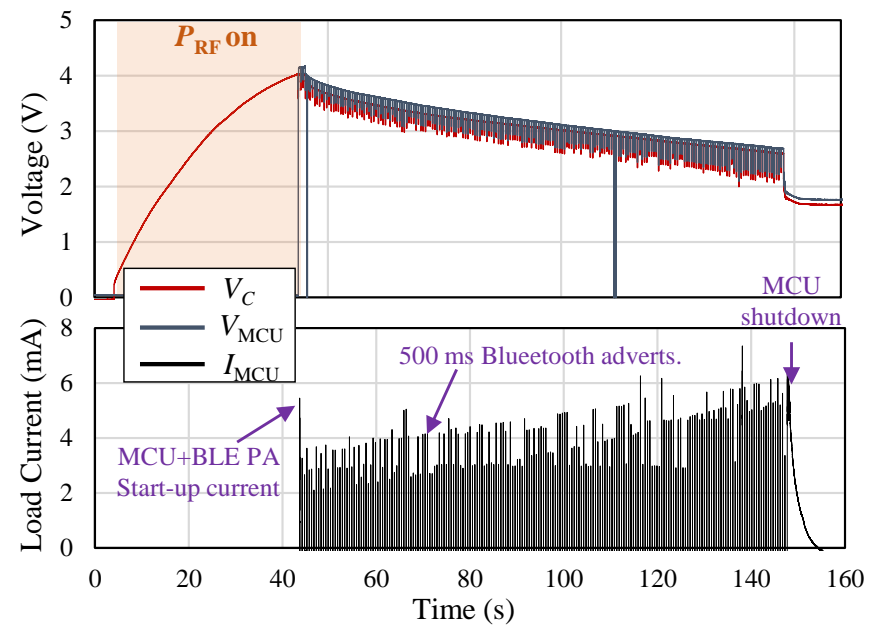

Fig. 5. Measuerd DC voltage across the supercapacitor, the SoC, and the current consumed by the BLE transmissions every $500 \mathrm{~ms}$.

unit (MCU) and a Bluetooth Low Energy (BLE) transceiver. While the measurements were performed using a development kit on a rigid PCB, a fully-flexible BLE node with a wearable antenna has previously been implemented based on the same module [15]. The BLE transmitter was programmed to transmit at $0 \mathrm{dBm}$, enabling a communication range of up to $100 \mathrm{~m}$ with a remotely-located off-body receiver, every $500 \mathrm{~ms}$. The SoC consumes around $6 \mathrm{~mA}$ during the active transmission period, based on the datasheet parameters.

The voltage across the supercapacitor and the MCU were monitored along with the current consumption (using a $10 \Omega$ current sense resistor) using a three-channel oscilloscope. Fig. 5 shows the textile supercapacitor's voltage charging up to $4 \mathrm{~V}$, before the cold-start circuit enables the MCU to turn on and transmit every $500 \mathrm{~ms}$, as observed in the current spikes measured through the resistor.

\section{CONCLUSION}

In this paper, the performance of a carbon-based organic electrolyte e-textile supercapacitor in RF energy harvesting applications was investigated. It was observed, through timevarying s-parameters, that despite the optimal rectifier matching with a resistive load, the variation in the charging current of a capacitor limits the maximum achievable efficiency of a rectifier. The proposed textile-based supercapacitor was then demonstrated for the first time directly powering a wireless Bluetooth sensor node, from a minimum input of $4 \mathrm{dBm}$, and for up to $102 \mathrm{~s}$ from $39 \mathrm{~s}$ of charging at $9.5 \mathrm{dBm}$. Based on the measured results, rectennas integrated with supercapacitors are highly suitable for powering BAN nodes.

\section{REFERENCES}

[1] M. Wagih, N. Hillier, S. Yong, A. S. Weddell, and S. Beeby, "Rfpowered wearable energy harvesting and storage module based on etextile coplanar waveguide rectenna and supercapacitor," IEEE Open Journal of Antennas and Propagation, vol. 2, pp. 302 - 314, 2021.

[2] G. Monti, L. Corchia, and L. Tarricone, "UHF Wearable Rectenna on Textile Materials," IEEE Trans. Antennas. Propag., vol. 61, 7, pp. 3869 - 3873, 2013.

[3] J. A. Estrada, E. Kwiatkowski, A. López-Yela, M. Borgoñós-García, D. Segovia-Vargas, T. Barton, and Z. Popović, "An RF-Harvesting Tightly-Coupled Rectenna Array Tee-Shirt with Greater than Octave Bandwidth,' IEEE Trans. Microw. Theory Techniq., vol. 68 no. 9, pp. 3908 - 3919, 2020.

[4] M. Wagih, G. S. Hilton, A. S. Weddell, and S. Beeby, "Broadband Millimetre-Wave Textile-based Flexible Rectenna for Wearable Energy Harvesting ," IEEE Trans. Microw Theory Techn, vol. 68 no. 11, pp. 4960 - 4972, 2020.

[5] S. A. Nauroze, J. G. Hester, B. K. Tehrani, W. Su, J. Bito, R. Bahr, J. Kimionis, and M. M. Tentzeris, "Additively Manufactured RF Components and Modules: Toward Empowering the Birth of Cost-Efficient Dense and Ubiquitous IoT Implementations," Proc. IEEE, vol. 105, no. 4, pp. $702-722,2017$

[6] J. Bito, R. Bahr, J. G. Hester, S. A. Nauroze, A. Georgiadis, and M. M. Tentzeris, "A Novel Solar and Electromagnetic Energy Harvesting System With a 3-D Printed Package for Energy Efficient Internet-ofThings Wireless Sensors," IEEE Trans. Microw. Theory Techn., vol. 65 no. 5, pp. $1831-1842,2017$.

[7] M. Wagih, G. S. Hilton, A. S. Weddell, and S. Beeby, "Dual-Band DualMode Textile Antenna/Rectenna for Simultaneous Wireless Information and Power Transfer (SWIPT)," IEEE Trans. Antennas Propag., 2021.

[8] M. Wagih, O. Cetinkaya, B. Zaghari, A. S. Weddell, and S. Beeby, "Real-World Performance of Sub-1 GHz and $2.4 \mathrm{GHz}$ Textile Antennas for RF-Powered Body Area Networks," IEEE Access, vol. 8, pp. 133746 - 133756, 2020.

[9] P. Li, Z. Long, and Z. Yang, "RF Energy Harvesting for BatteryLess and Maintenance-Free Condition Monitoring of Railway Tracks," IEEE Internet of Things Journal, vol. Early Access, p. DOI: 10.1109/JIOT.2020.3023475, 2020.

[10] A. Okba, D. Henry, A. Takacs, and H. Aubert, "Autonomous RFID Sensor Node Using a Single ISM Band for Both Wireless Power Transfer and Data Communication," Sensors, vol. 19 (15), p. 3330, 2019.

[11] W. Zhao, K. Choi, S. Bauman, Z. Dilli, T. Salter, and M. Peckerar, "A radio-frequency energy harvesting scheme for use in low-power ad hoc distributed networks," IEEE Trans. Circuits And Systems, vol. 59 no. 9, pp. 573 - 577, 2012.

[12] N. Hillier, S. Yong, A. Cruden, and S. Beeby, "Acetonitrile-free organic electrolyte for textile supercapacitor applications," Journal of The Electrochemical Society, 2021.

[13] S. Yong, J. Shi, and S. Beeby, "Wearable Textile Power Module Based on Flexible Ferroelectret and Supercapacitor," Energy Technology, vol. 7 (5), pp. $2284-2291,2018$.

[14] M. Wagih, A. S. Weddell, and S. Beeby, "Omnidirectional DualPolarized Low-Profile Textile Rectenna with over 50\% Efficiency for Sub- $\mu \mathrm{W} / \mathrm{cm}^{2}$ Wearable Power Harvesting," IEEE Transactions on Antennas and Propagation, vol. 69, no. 5, pp. 2522-2536, 2021.

[15] M. Wagih, Y. Wei, and S. Beeby, "Flexible $2.4 \mathrm{GHz}$ Sensor Node for Body Area Networks with a Compact High-Gain Planar Antenna," IEEE Antennas Wireless Propag. Lett., vol. 17, 12, pp. 49 - 53, 2018. 